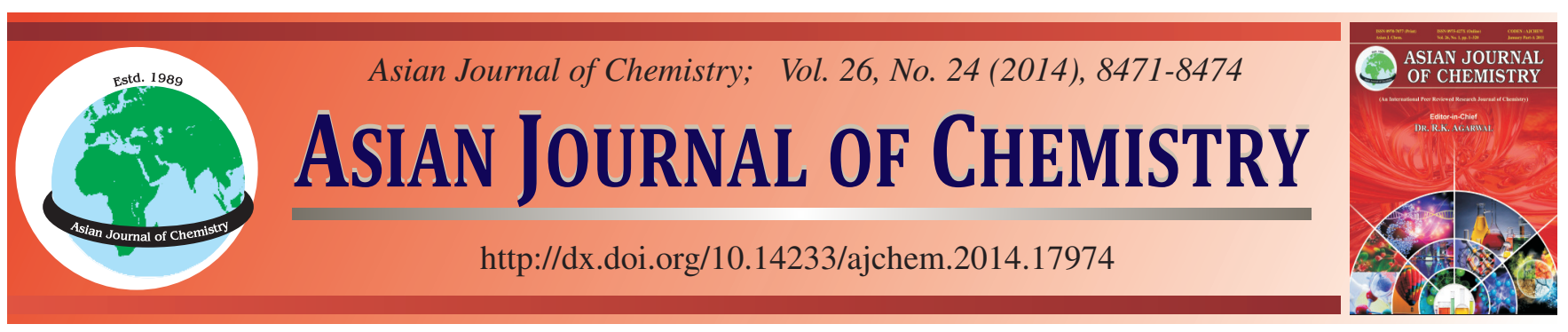

\title{
Mechanical Studies on Chitosan Functionalized Multiwalled Carbon Nanotube Reinforced Epoxy Bionanocomposites
}

\author{
V. Selvam ${ }^{1, *}$, M. Suresh Chandra Kumar ${ }^{1}$, S. Julyes Jaisingh ${ }^{2}$ and M. VAdivel ${ }^{1}$
}

${ }^{1}$ Polymer Nanocomposite Centre, Department of Chemistry and Research Centre, Scott Christian College, Nagercoil-629 003, India ${ }^{2}$ Department of Mechanical Engineering, St. Xavier's Catholic College of Engineering, Nagercoil-629 003, India

*Corresponding author: E-mail: selvam.che@gmail.com

Received: 9 May 2014;

Accepted: 16 August 2014;

Published online: 1 December 2014;

AJC-16384

\begin{abstract}
The chitosan functionalized multiwalled carbon nanotubes reinforced epoxy bionanocomposite (CS- $g$-MWCNTs/EP) was developed. Chitosan (CS) was grafted to multiwalled carbon nanotubes (MWCNTs), first by reacting the oxidized carbon nanotubes (MWCNT$\mathrm{COOH}$ ) with thionyl chloride to form acyl-chlorinated multiwalled carbon nanotubes (MWCNT-COCl) followed by subsequent dispersion in chitosan. The chitosan grafted multiwalled carbon nanotubes (CS- $g$-MWCNTs) were reinforced in the epoxy matrix using high speed stirrer and then cured. The TEM of CS- $g$-MWCNTs shows a visible coating of chitosan on the surface of MWCNTs. FT-IR spectra of CS-g-MWCNTs indicate an overlap of the amide band and the free amino groups of the chitosan. The mechanical properties such as tensile and impact strength of epoxy matrix increases to 16 and $52 \%$, respectively by the reinforcement of 1.5 wt. \% CS- $g$-MWCNTs, which clearly reveals that the free amino groups of CS- $g$-MWCNTs acted as a curing agent and was covalently attached into epoxy matrix. Morphologies of pure chitosan, MWCNT, MWCNT-COOH and CS- $g$-MWCNTs/EP bionanocomposites were studied by scanning electron microscope.
\end{abstract}

Keywords: Chitosan, Multiwalled carbon nanotubes, Bionanocomposites, Mechanical properties, Morphology.

\section{INTRODUCTION}

Organic light emitting diodes and organic photovoltaics are susceptible to chemical degradation in the presence of oxygen and moisture. The sensitivity of these materials towards oxygen and moisture makes it imperative to protect them by encapsulation. High quality epoxy resins, having exceptional combination of properties such as toughness, adhesion and chemical resistance ${ }^{1}$, are used for effective fixture and encapsulation of the electronic components. The epoxy encapsulated electronic parts are protected from adverse environmental, mechanical, thermal and electrical stresses. Carbon nanotubes (CNTs) have unique molecular structures and outstanding thermal, electronic, optical and mechanical properties ${ }^{2-6}$. Furthermore, they are potentially useful fillers in nanocomposites with polymers, metals and ceramics ${ }^{7-10}$. Addition of conductive fillers in an insulating matrix dramatically increases the electrical properties when a network was developed throughout the matrix. The increase in electrical properties of the composites depends on the property of the conducting fillers, dispersion of conducting fillers in polymeric matrix and interaction between the fillers and the polymer ${ }^{11-14}$. The incorporation of CNTs into polymer bulk materials will surely enhance their thermal and electrical properties ${ }^{15}$. An enhancement of the compatibility between the CNTs and resin matrix can improve the electrical and mechanical properties of the composite materials. Chitosan is a natural polymer generally obtained by extensive deacetylation of chitin and has many industrial and pharmaceutical applications ${ }^{16}$. Decorating CNTs with nonconducting natural polymers will decrease its electrical conductance. Further, reinforcement of biopolymer grafted CNTs onto polymer matrix will undergo degradation easily.

In this work chitosan grafted MWCNTs (CS- $g$-MWCNTs) and CS- $g$-MWCNTs/EP bionanocomposites were developed and their structural, mechanical, thermal and morphological properties were investigated.

\section{EXPERIMENTAL}

Commercially available diglycidyl ether of bisphenol-A based epoxy resin (DGEBA), LY556, having epoxy equivalent $=180-190$, with viscosity $=10,000 \mathrm{cP}$ and 4,4'-diamino-diphenylmethane (DDM), epoxy curing agent were obtained from CibaGeigy Ltd., India. Multiwalled carbon nanotubes (MWCNTs) with surface area $=200 \mathrm{~m}^{2} / \mathrm{g}$, diameter $=10-20 \mathrm{~nm}$, length $>$ $10 \mu$ and purity $>98 \%$ was purchased from Chemapol Industries Ltd, India. Chitosan having molecular mass $(\mathrm{Mv})=1,80,000$ $\mathrm{Da}$, moisture content $=9.46 \%$, viscosity $=229 \mathrm{cP}, \mathrm{DD}=86.39$ was provided by Indian sea foods, Cochin, India. 
Purification of chitosan: $10 \mathrm{~g}$ of chitosan was dissolved in $200 \mathrm{~mL}$ of $1 \%$ acetic acid solution, then filtered, using glass wool to remove insoluble sample. $200 \mathrm{~mL}$ of $2 \mathrm{M}$ sodium hydroxide was added to the filtered sample with continuous stirring. After removal of excess $\mathrm{NaOH}$ solution, the filtrate was washed with distilled water until a $\mathrm{pH} 7$ was reached. The structure of chiotsan is shown in Fig. 1.

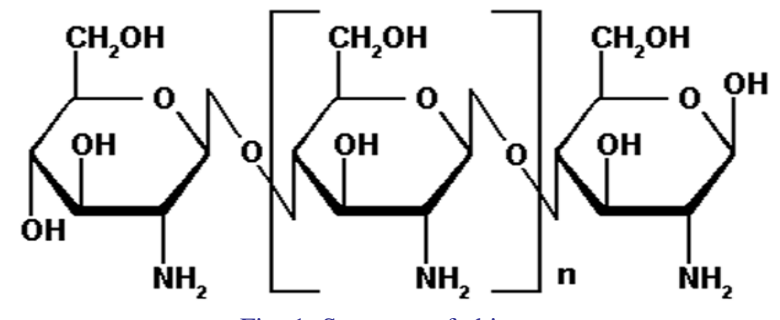

Fig. 1. Structure of chitosan

Preparation of CS-g-MWCNTs composites: $2 \mathrm{~g}$ of MWCNTs was dissolved in $100 \mathrm{~mL}$ conc. $\mathrm{HCl}$ for the removal of metal impurities. Then it was washed with distilled water until become neutral. Multiwalled carbon nanotubes were functionalized with carboxyl group by sonication at $40{ }^{\circ} \mathrm{C}$ for $2 \mathrm{~h}$ with a $3: 1(\mathrm{v} / \mathrm{v})$ acid mixture $\left(98 \% \mathrm{H}_{2} \mathrm{SO}_{4}\right.$ and $\left.65 \% \mathrm{HNO}_{3}\right)$. The oxidized carbon nanotubes (MWCNT-COOH) were suspended in a solution of thionyl chloride and refluxed for $24 \mathrm{~h}$ at $75^{\circ} \mathrm{C}$ to convert carboxyl groups to acyl chloride groups. The acyl chloride functionalized carbon nanotubes (MWCNT$\mathrm{COCl}$ ) were separated by membrane filtration, washed with THF and dried in an oven. Chitosan was mixed with MWCNT$\mathrm{COCl}$ in DMF and refluxed at $120^{\circ} \mathrm{C}$ for $96 \mathrm{~h}$ under nitrogen atmosphere. After reaction, the mixture was filtered through a $0.22 \mu \mathrm{m}$ polyether sulphone membrane and dried in a vacuum oven to obtain black chitosan grafted MWCNT (CS- $g$-MWCNTs) composite powder ${ }^{17}$. Fig. 2 represents the formation of CS- $g$ MWCNTs.
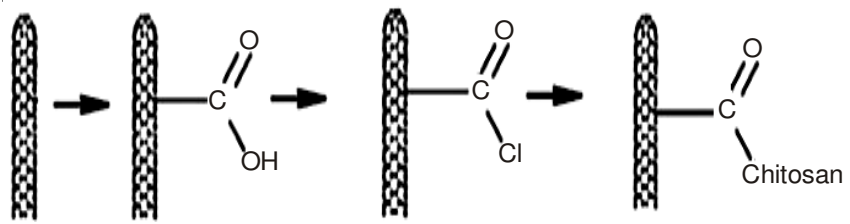

Fig. 2. Schematic representation of chitosan grafted MWCNTs

Preparation of neat epoxy matrix: $100 \mathrm{~g}$ of epoxy resin (DGEBA) was heated to $70{ }^{\circ} \mathrm{C}$ in an oil bath for the removal of trapped air bubbles and moisture. The amine hardener (DDM) (27\% in accordance with the epoxy resin) was also heated to $90{ }^{\circ} \mathrm{C}$ to melt completely. Both the resin and hardener were mixed with continuous stirring and then poured into mould, cured in an air oven at $100{ }^{\circ} \mathrm{C}$ for $4 \mathrm{~h}$ and post cured at $140{ }^{\circ} \mathrm{C}$ for $3 \mathrm{~h}$.

Preparation of CS-g-MWCNTs/epoxy bionanocomposites: Calculated amount of CS- $g$-MWCNTs $(0.1,0.5,1.0$, $1.5,2.0 \mathrm{~g}$ ) were added to the epoxy resin and the mixture was sonicated for $2 \mathrm{~h}$ followed by continuous stirring for $20 \mathrm{~min}$. at $12000 \mathrm{rpm}$. The resin mixture, benzoyl peroxide and the hardener (DDM) were mixed with continuous stirring and then cured and post cured in an air oven.
Analytical methods: FT-IR spectra of MWCNTs, MWCNT$\mathrm{COOH}$, chitosan and CS- $g$-MWCNTs were recorded on a Perkin-Elmer Spectrometer in the range of $4000-400 \mathrm{~cm}^{-1}$ at room temperature. The thermal analysis was carried out by a Du Pont Differential Scanning Calorimeter with a heating rate of $10^{\circ} \mathrm{C} / \mathrm{min}$. Tensile strength was determined as per ASTMD3039 using a universal testing machine (Instron, Model 6025 $\mathrm{UK}$ ), at a cross-head speed of $10 \mathrm{~mm} / \mathrm{min}$. with specimens of width $25 \mathrm{~mm}$, length $200 \mathrm{~mm}$ and thickness $3 \mathrm{~mm}$. Unnotched Impact strength was investigated as per ASTM-D256-88 using Izod impact tester with specimens of $3 \mathrm{~mm}$ thickness, $10 \mathrm{~mm}$ width and $90 \mathrm{~mm}$ length. For both studies five specimens were tested for each sample. Transmission Electron Microscopy was recorded using TEM 100CX (JEOL) with an accelerating voltage of $100 \mathrm{keV}$ to find the size and shape of MWCNTs and CS- $g$-MWCNTs. Surface morphology of fractured surface of the composites were recorded using scanning electron microscope (SEM; JEOL JSM Model 6360).

\section{RESULTS AND DISCUSSION}

FT-IR spectroscopy: FT-IR spectroscopy was used to characterize the chemical components on the MWCNTs surface and the result shown in Fig. 3. Although there were no characteristics peaks in the FT-IR spectra of MWCNTs [Fig. 3(a)], the MWCNT-COOH shows several vibrational peaks, carboxylic $\mathrm{C}=\mathrm{O}$ stretching vibration at $1635 \mathrm{~cm}^{-1}$, O-H stretching vibration at $3440 \mathrm{~cm}^{-1}$ and $\mathrm{C}-\mathrm{H}$ stretching and bending vibrations at 2889, 1209 and $1149 \mathrm{~cm}^{-118}$. These results indicate that carboxylic acid groups have been covalently attached to the surface of the MWCNTs [Fig. 3(b)].

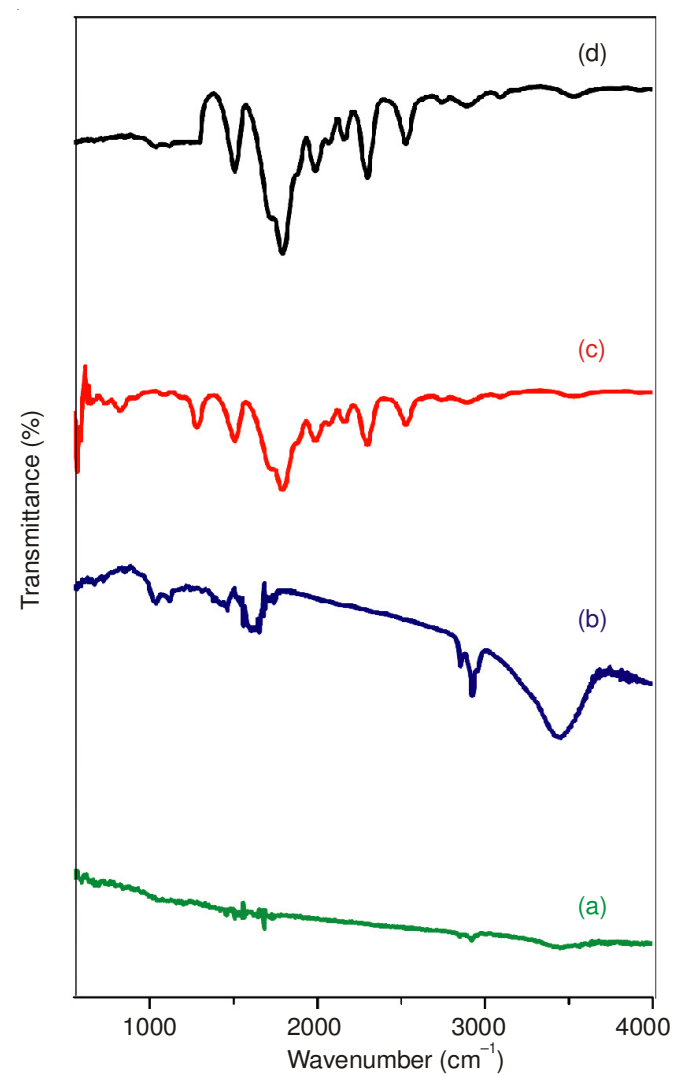

Fig. 3. FT-IR of (a) MWCNTs, (b) MWCNT-COOH, (c) Chitosan, (d) CS$g$-MWCNT 
FT-IR spectra of chitosan: Fig. 3(c) represents the following: $\mathrm{C}=\mathrm{O}$ stretching vibration of acetyl unit at $1647 \mathrm{~cm}^{-1}$, $\mathrm{C}-\mathrm{O}-\mathrm{C}$ stretching vibration at $1091 \mathrm{~cm}^{-1}, \mathrm{~N}-\mathrm{H}$ bending vibration at $1585 \mathrm{~cm}^{-1}$ and $\beta(1,4)$ glycosidic bond vibration at $1151 \mathrm{~cm}^{-1}$. The broad peak at $3431 \mathrm{~cm}^{-1}$ due to $-\mathrm{OH}$ stretching vibration is superimposed on -NH stretching band and broaden due to inter hydrogen bonds of polysaccharides.

The FT-IR of CS-g-MWCNTs is shown in Fig. 3(d). When compared to MWCNT-COOH Fig. 3(b) a new peak due to stretching vibrations of $-\mathrm{OH}$ and $-\mathrm{NH}$ is obtained at $3430 \mathrm{~cm}^{-1}$. Additionally a new absorption peak at $1586 \mathrm{~cm}^{-1}$ indicates an overlap of the amide band and the amino group of the chitosan $^{19,20}$. Similarly, peaks due to $\beta(1,4)$ glycosidic bonds and C-O-C stretching vibration are shifted to 1123 and 1041 $\mathrm{cm}^{-1}$, respectively. The above results confirm that chitosan is grafted on the MWCNTs surfaces.

Mechanical properties: The observed values for tensile and unnotched impact properties of the CS- $g$-MWCNTs reinforced epoxy bionanocomposites are shown in Figs. 4 and 5. Fig. 4 shows that the tensile strength of the bionanocom-posites increases from 72.5 M.Pa (neat epoxy) to $87.54 \mathrm{M} . \mathrm{Pa}$ (1.5 wt. $\%$ CS-g-MWCNTs). Fig. 5 shows the unnotched impact

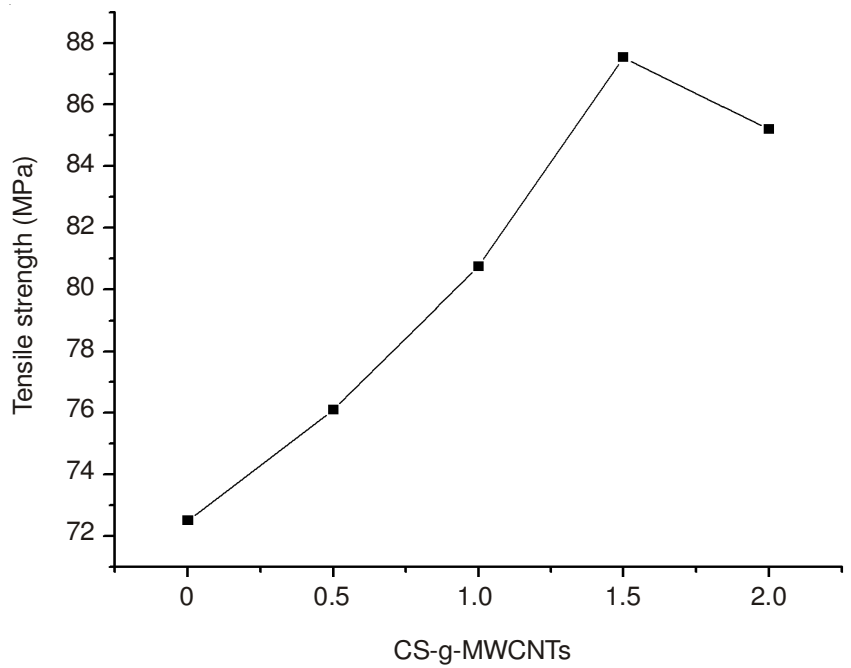

Fig. 4. Effect of CS- $g$-MWCNTs on the tensile strength of CS- $g$-MWCNTs/ EP bionanocomposites

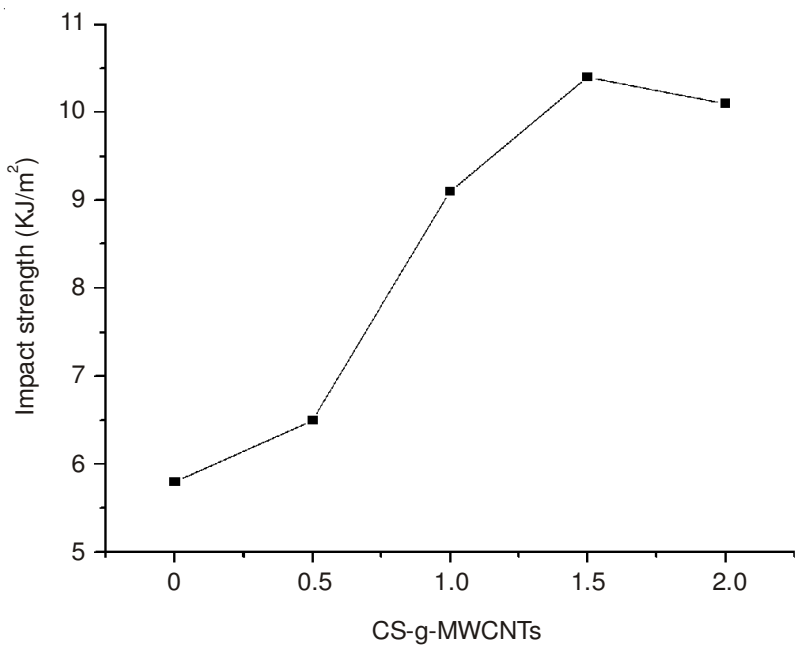

Fig. 5. Effect of CS-g-MWCNTs on the impact strength of CS-gMWCNTs/EP bionanocomposites strength of bionanocomposites increases from $5.6 \mathrm{KJ} / \mathrm{m}^{2}$ (neat epoxy) to $10.5 \mathrm{KJ} / \mathrm{m}^{2}$ (1.5 wt. \% CS- $g$ MWCNTs). The significant improvement in mechanical properties is due to the formation of covalent bond between free amine group of CS$g$-MWCNTs and epoxy matrix which leads to effect stress transfer. However, as CS- $g$-MWCNTs content increases to $2 \mathrm{wt} . \%$, the tensile strength and impact strength decreases slightly due to agglomeration.

TEM analysis: TEM image of CS- $g$-MWCNTs (Fig. 6b) shows the immobilization of chitosan on the surface of MWCNTs and is quite visible when compared to TEM of pure MWCNTs (Fig. 6a). This is the clear indication of functionalization of chitosan on the surface of MWCNTs.

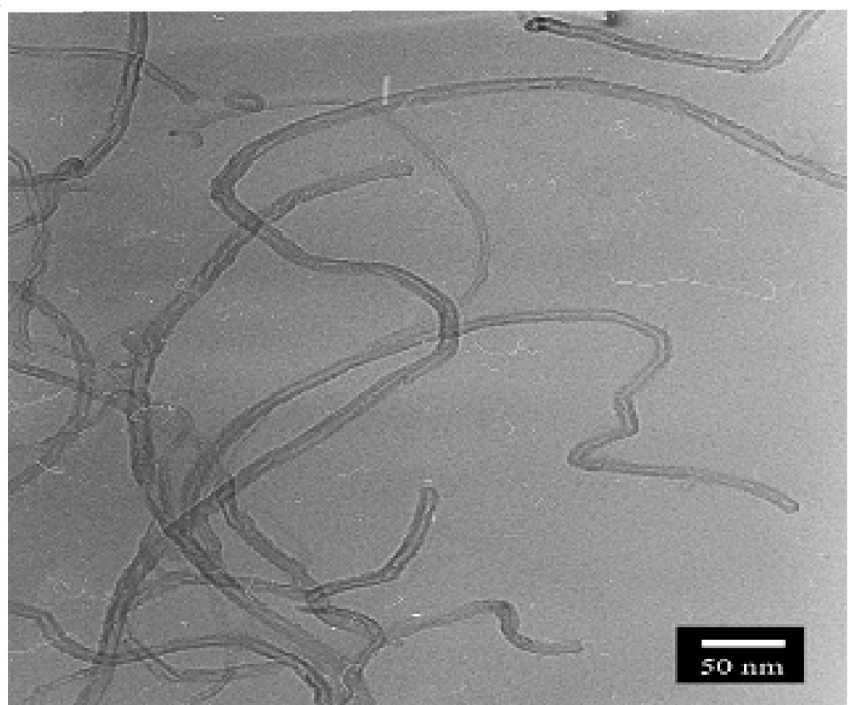

Fig. 6. (a) TEM image of MWCNTs

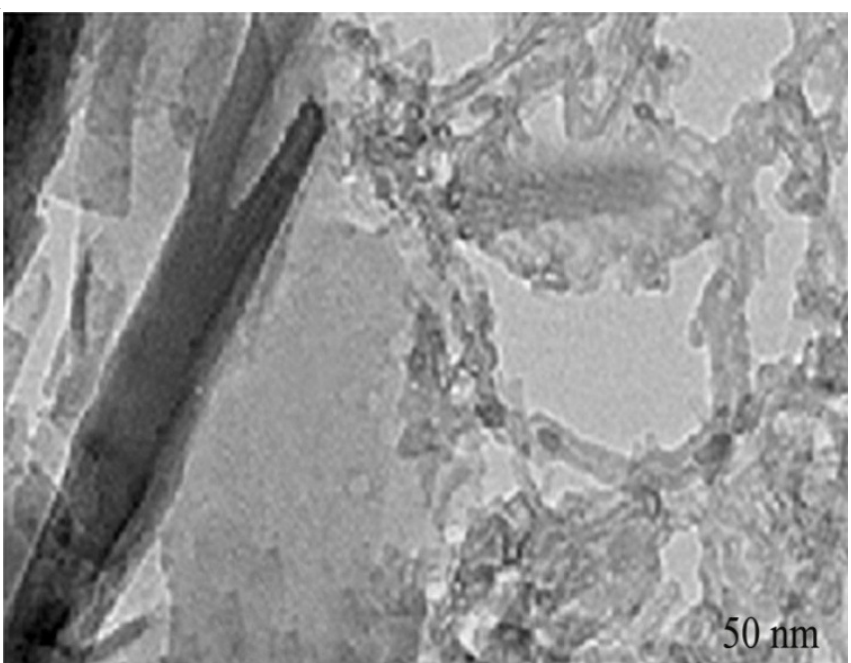

Fig. 6. (b) TEM image of CS-g-WCNTs

SEM analysis: SEM micrograph of unmodified MWCTs (Fig. 7a) reveals a clean and smooth surface with bundles. In contrast, the SEM of MWCNT-COOH (Fig. 7b) shows separation of bundles.

Fig. 7c and 7d shows the SEM images of tensile fracture surfaces for MWCNT-COOH/EP composites and CS- $g$-MW$\mathrm{CNTs} / \mathrm{EP}$ bionanocomposites, respectively. The fracture surface of MWCNT-COOH reinforced epoxy nanocomposites indicates 


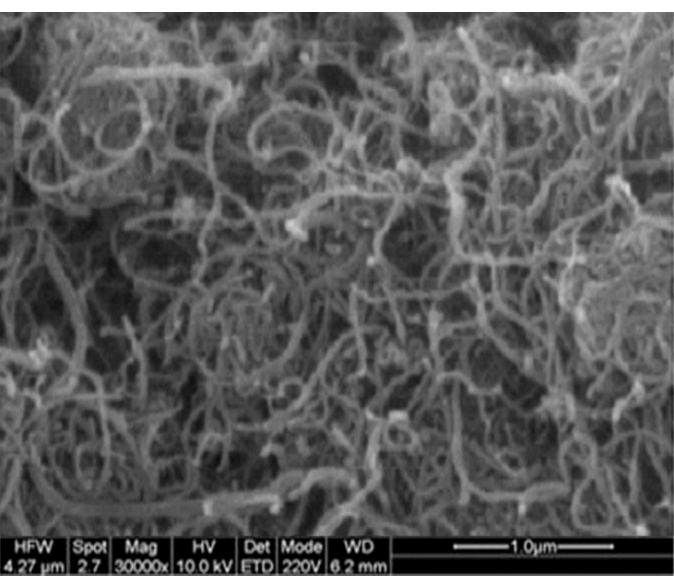

Fig. 7. (a) SEM of unmodified MWCNTs

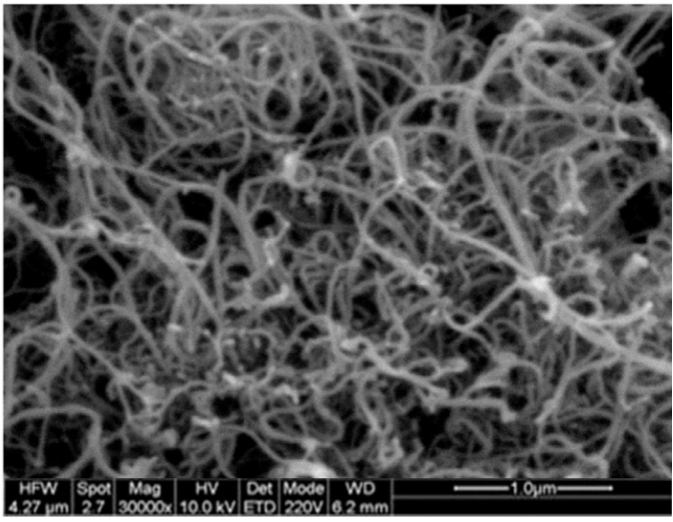

Fig. 7. (b) SEM of MWCNT-COOH

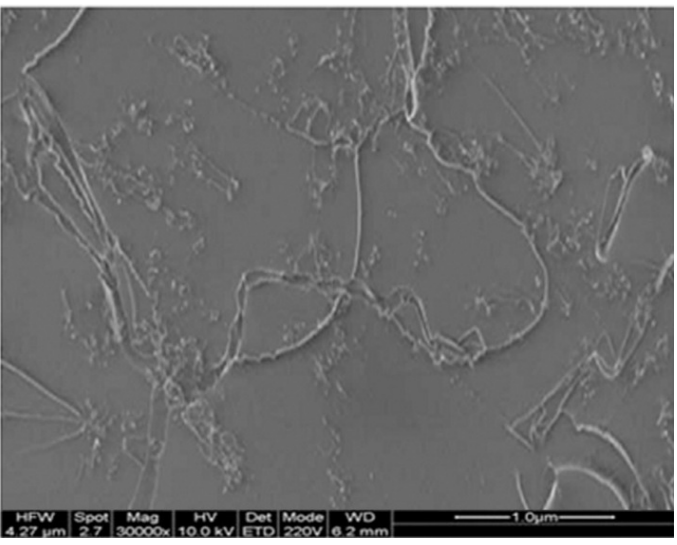

Fig. 7. (c) SEM of MWCNT-COOH/EP

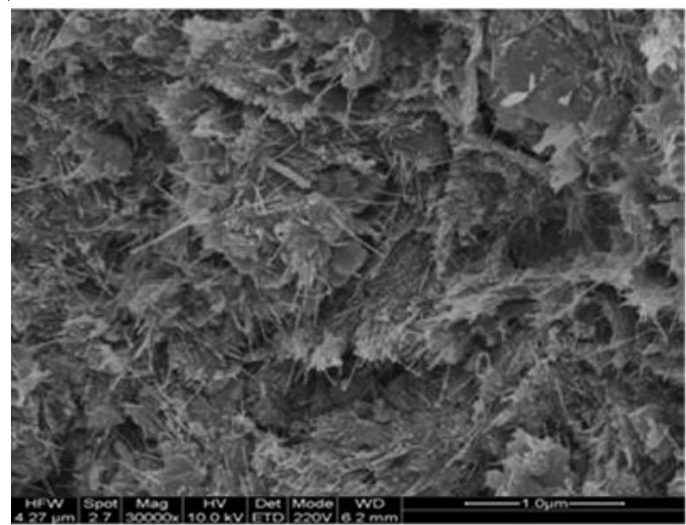

Fig. 7. (d) SEM of CS-g-MWCNTs/EP the well dispersion of nanoparticles in epoxy matrix ${ }^{21}$. CS- $g$ MWCNTs filled epoxy bionanocomposites shows the reinforcement of CS- $g$-MWCNTs flakes in epoxy matrix.

\section{Conclusion}

The chitosan was successfully functionalized onto MWCNTs a surface which was confirmed by FT-IR and TEM. Increase in mechanical and thermal properties of CS- $g$ MWCNTs/EP bionanocomposites is due to complicated reaction between free amino groups of CS- $g$-MWCNTs with epoxy matrix. The fracture surfaces of CS-g-MWCNTs/EP bionanocomposites indicate the dispersion of CS- $g$-MWCNTs onto epoxy matrix. This bionanocomposites can be used for the encapsulation of electronic devices such as organic light emitting diodes and organic photovoltaics which are susceptible to chemical degradation in the presence of oxygen and moisture. Further, CS- $g$-MWCNTs/EP bionanocomposites are degradable and environment friendly.

\section{REFERENCES}

1. K.S. Meenakshi and E.P.J. Sudhan, Arabian J. Chem., doi:10.1016/ j.arabjc.2011.05.020.

2. Q. Gong, Z. Li, X. Bai, D. Li, Y. Zhao and J. Liang, Mater. Sci. Eng. A, 384, 209 (2004).

3. Q. Huang, L. Gao, Y. Liu and J. Sun, J. Mater. Chem., 15, 1995 (2005).

4. J. Hone, C. Llaguno, M. Nemes, T. Johnson, E. Fischer, A. Walters, M.J. Casavant, J. Schmidt and R.E. Smalley, Appl. Phys. Lett., 77, 666 (2000).

5. P.J. de Pablo, E. Graugnard, B. Walsh, R.P. Andres, S. Datta and R. Reifenberger, Appl. Phys. Lett., 74, 323 (1999).

6. F. Lin, F. Shyu and R. Chen, Phys. Rev. B Condens. Matter, 61, 14114 (2000).

7. C. Park, J. Wilkinson, S. Banda, Z. Ounaies, E. Wise, G. Sauti, T. Lillehei and S. Harrison, J. Polym. Sci., B, Polym. Phys., 44, 1751 (2006).

8. H. Yun, V. Shanov, J. Schulz, S. Narasimhadevara, S. Subramaniam, D. Hurd and F.J. Boerio, Smart Mater. Struct., 14, 1526 (2005).

9. S. Guo, S. Dong and E. Wang, Small, 4, 1133 (2008).

10. G. Chai, Y. Sun, J. Sun and Q. Chen, J. Micromech. Microeng., 18, 35013 (2008).

11. Y. Huang, N. Li, Y. Ma, F. Du, F. Li, X. He, X. Lin, H. Gao and Y. Chen, Carbon, 45, 1614 (2007).

12. F. Dalmas, R. Dendievel, L. Chazeau, Y. Cavaille and C. Gauthier, Acta Mater., 54, 2923 (2006).

13. H. Yao, M. Dang, J. Jiang and P. Xu, Appl. Phys. Lett., 91, 212901 (2007).

14. J. Sandler, P. Shaffer, T. Prasse, W. Bauhofer, K. Schulte and H. Windle, Polymer, 40, 5967 (1999).

15. H. Gojny, H. Wichmann, B. Fiedler, A. Kinloch, W. Bauhofer, A.H. Windle and K. Schulte, Polymer, 47, 2036 (2006).

16. M.N.V.R. Kumar, R.A.A. Muzzarelli, C. Muzzarelli, H. Sashiwa and A.J. Domb, Chem. Rev., 104, 6017 (2004).

17. M.F. Alkhatib, M.E.S. Mirghani, I.Y. Qudsieh and I.A.F. Husain, J. Appl. Sci., 10, 2705 (2010).

18. J.F. Chen, J. Wang, M. Lin, W. Zhong, T. Feng, X. Chen, J. Chen and F. Xue, Mater. Sci. Eng. A, 492, 236 (2008).

19. L. Carson, C. Kelly-Brown, M. Stewart, A. Oki, G. Regisford, Z. Luo and V.I. Bakhmutov, Mater. Lett., 63, 617 (2009).

20. A.R. Cestari, E.F.S. Vieira, F.J. Alves, E.C.S. Silva and M.A.S. Andrade Jr., J. Hazard. Mater., 213, 109 (2012).

21. M.-K. Yeh, T.-H. Hsieh and N.-H. Tai, Mater. Sci. Eng. A, 483-484, 289 (2008) 